\title{
Study on Absorption of VOCs into PDMS Film using Heterodyne Interferometry for Application of VOCs Sensing.
}

\author{
Sulaiman Khan ${ }^{1,3}$, David Newport ${ }^{1}$, Stephané Le Calvé2,3 \\ ${ }^{1}$ Department of Mechanical Aeronautical \& Biomedical Engineering, Bernal Institute, University of \\ Limerick Ireland, \\ 2University of Strasbourg, Institute of Chemistry and Processes for Energy, Environment and Health \\ (ICPEES), Group of Atmospheric Physical Chemistry, Strasbourg France. \\ 3In'Air Solutions, Strasbourg France. \\ David.newport@ul.ie
}

\begin{abstract}
A heterodyne interferometer using a Mach-Zehnder optical configuration is designed for detection of airborne VOCs. The design is based on film-mediated sensing technique in which the response of intermediate sensing film is monitored using interferometry. PDMS is used as a sensing film, which changes its volume and refractive index when absorbed VOCs molecules. Thin film of PDMS acts as a phase object and any variation in its state causes a proportional phase shifts in the transmitted light beam. The Mach-Zehnder interferometer is integrated with a microscope and acoustic-optic modulators. The information of the absorbed gas concentration into the film are encoded in the fringe patterns and are extracted by a temporal phase extraction heterodyne method. The film is exposed to different concentrations of air-borne toluene and its response is measured using interferometry.
\end{abstract}

Key words: VOCs, BTEX, Mach-Zehnder Interferometer, Optical sensor, film-mediated sensor

Monitoring of indoor air quality is important for healthy living. On average a person spends about $90 \%$ daily time in indoor environments where exposure to various indoor air pollutants like volatile organic compounds (VOCs) is inevitable [1]. Some of VOCs i.e. benzene, toluene, ethylbenzene and xylene (BTEX) are harmful and long-term exposure may pose a serious threat to human health. Among BTEX, benzene is identified as carcinogenic [2]. The monitoring of VOCs at sub-ppb level requires an ultra-sensitive technique. Interferometry based detection can be a viable option for detecting VOCs at ppb level.

Interferometry based sensing techniques are ultra-sensitive and have been employed for pressure, temperature and concentration measurements in microchannels [3]. Its application has been extended to gas detection by employing a VOCs sensitive film. In previous work, the VOCs sensing using interferometry is limited to ppm level. Martinex et al. used a Pohl interferometry setup for VOCs detection with a sensitivity of 1500 ppm [4]. Reddy et al. used FP interferometer integrated with $\mu \mathrm{GC}$ and detected toluene down to 25 ppb [5]. Kacik et al. developed optical fiber sensor by using a micro cavity in PDMS and detected toluene with a sensitivity of $0.15 \mathrm{~nm} / \mathrm{g}-\mathrm{m}^{-3}$ [6]. In this study, we investigated the application of Mach-Zehnder interferometer configured with heterodyning for detection of air-borne toluene. The integration of heterodyning with interferometer enhance its sensitivity. This technique is capable to retrieve nanometric level variations (40nm) [7].

The optical setup is designed based on MachZehnder interferometer with heterodyning. A light from a coherent He-Ne laser $(\lambda=633 \mathrm{~nm}$, $35 \mathrm{~mW}$, JDS Uniphase) source is guided by a single mode optical fiber. The light beam is split into two beams by 50:50 fiber coupler (BS1). One beam acts as a test beam while the other acts as a reference beam. An inverted optical microscope (Olympus IX41) is configured with the test beam through a fiber from the BS1 fiber coupler. The collimating lens allows a planar light wavefront to transverse the sample. The phase is same all across the plane and a proportional variation occurs while traversing the sample. Microscope objective collects the wavefront passing the sample which then interferes with the reference beam at the cubical beam splitter 
(BS). The setup is shown in the Fig.1 (A). The reference arm acts as a wavefront shaping optical arrangement which can be used to control the number and shape of fringes on the camera. The interferograms are captured by high speed CMOS camera and then analyzed using homemade LabVIEW program.

A polydimethylsiloxane (PDMS) is used as a toluene sensing film, which changes its volume and refractive index when expose to toluene [8]. PDMS are silicone-based elastomer and are widely used in microfluidics applications due to its features like optical transparency, chemical inertness, bio-compatibility, low cost, flexibility, reversible deformation in mechanical stress and tunable permeability [9]. Although the swelling of PDMS is not desired for many application however it can be exploited for the actuation mechanism in sensing devices [8]. PDMS is shaped into a rectangular slab with thickness of $100 \mu \mathrm{m}$ and $500 \mu \mathrm{m}$ and enclosed in a small chamber. The gas chamber has size $25 \mathrm{~mm} x$ $25 \mathrm{~mm} \times 25 \mathrm{~mm}$. Top and bottom are covered with glass to allow the light beam to pass through the PDMS film. The gas is flowing through the sides and the light beam is passing through PDMS from top to bottom as shown in Fig.1 (B). Highly precise mass flow controller (accuracy $<0.5 \%$ ) are used to generate the desired dosage of toluene. The concentration of toluene down to $\mathrm{ppb}$ is generated with precision of $10 \%$.

Heterodyning is realized with a pair of fiber coupled up-shifted Acoustic-Optic Modulator (AOM) in each arm of the interferometer as shown in Fig.1 (A). AOMs employ sound waves to diffract the light beam and shift its frequency. The AOMs introduce frequency shift and provide a linear phase shift for temporal phase extraction. The swelling of PDMS, $\boldsymbol{\Delta} \boldsymbol{h}$ is calculated by using the phase information as given

$$
\Delta h=\frac{\lambda \varphi}{2 \pi n}
$$

respectively. Experiments using different flow rate and concentration are designed to investigate the absorption of VOCs into PDMS. The developed Mach-Zehnder interferometer integrated with microscope and heterodyning is highly sensitive and has potential to detect VOCs at sub-ppb level.

\section{Acknowledgements}

This ITN Research Project, MIGRATE is supported by European Community H2020 Framework under the Grant Agreement No. 643095 (H2020-MSCA-ITN-2014).

\section{References}

[1] N. E. Klepeis et al., "The National Human Activity Pattern Survey (NHAPS) A Resource for Assessing Exposure to Environmental Pollutants," 2001.

[2] D. Pyatt and S. Hays, "A review of the potential association between childhood leukemia and benzene," Chemico-Biological Interactions, vol. 184, no. 1-2. pp. 151-164, 19-Mar-2010.

[3] D. Newport at. al "Digital interferometry: techniques and trends for fluid measurement," Heat Mass Transf., vol. 44, no. 5, pp. 535-546, Mar. 2008.

[4] C. Martínez-Hipatl et al., "Detection of volatile organic compounds by an interferometric sensor," Sensors Actuators, B Chem., vol. 147, no. 1, pp. 37-42, 2010.

[5] K. Reddy et al., "Rapid, sensitive, and multiplexed on-chip optical sensors for micro-gas chromatography," Lab Chip, vol. 12, p. 901, 2012.

[6] D. Kacik and I. Martincek, "Toluene optical fibre sensor based on air microcavity in PDMS," Opt. Fiber Technol., vol. 34, pp. 70-73, Mar. 2017.

[7] S. Joseph et al., "A heterodyne Mach-Zehnder Interferometer employing static and dynamic phase demodulation techniques for live-cell imaging," 2010, vol. 7554, p. 75540P.

[8] C. V. Rumens et al., "Swelling of PDMS networks in solvent vapours; applications for passive RFID wireless sensors," J. Mater. Chem. C, vol. 3, no. 39, pp. 10091-10098, Oct. 2015.

[9] G. M. Whitesides et al., "Soft Lithography in Biology and Biochemistry," Annu. Rev. Biomed. Eng., vol. 3, no. 1, pp. 335-373, 2001.

Where $\lambda, \varphi$ and $n$ represents the light wavelength, phase and reflective index of PDMS

(A)

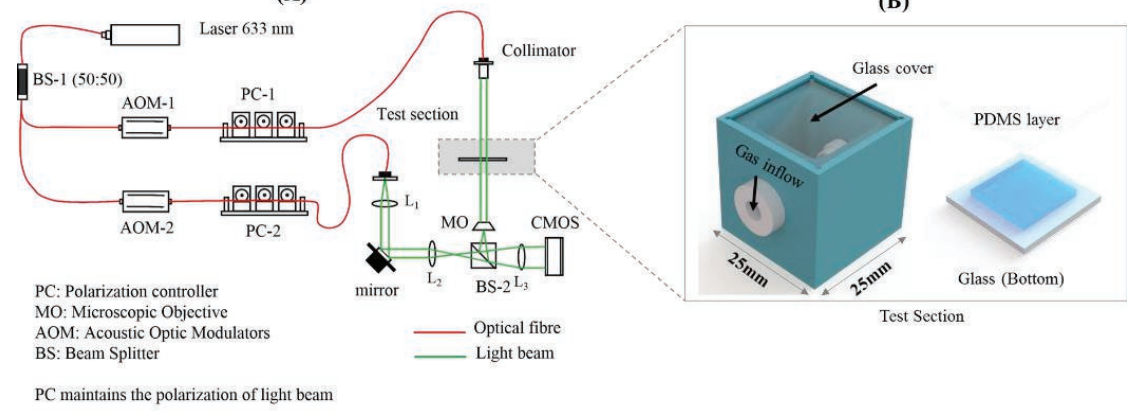

Figure 1. (A) Heterodyne interferometer configuration. AOMs are connected with a radio frequency driver and introduce frequency shift. (B) Micro gas chamber contain PDMS film. The chamber is $25 \mathrm{~mm} \times 25 \mathrm{~mm} \times 25 \mathrm{~mm}$. Top and bottom are covered with glass. 